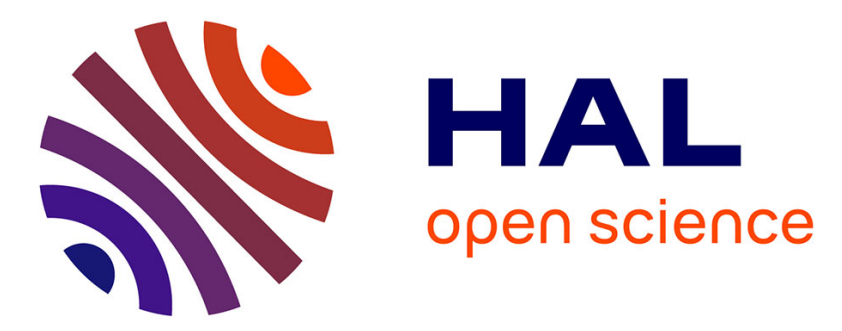

\title{
Computational determination of the dominant triplet population mechanism in photoexcited benzophenone
}

Dumitru-Claudiu Sergentu, Rémi Maurice, Remco W. A. Havenith, Ria Broer, Daniel Roca-Sanjuán

\section{- To cite this version:}

Dumitru-Claudiu Sergentu, Rémi Maurice, Remco W. A. Havenith, Ria Broer, Daniel Roca-Sanjuán. Computational determination of the dominant triplet population mechanism in photoexcited benzophenone. Physical Chemistry Chemical Physics, 2014, 16, pp.25393-25403. 10.1039/C4CP03277B . in2p3-01080854

\section{HAL Id: in2p3-01080854 https://hal.in2p3.fr/in2p3-01080854}

Submitted on 6 Nov 2014

HAL is a multi-disciplinary open access archive for the deposit and dissemination of scientific research documents, whether they are published or not. The documents may come from teaching and research institutions in France or abroad, or from public or private research centers.
L'archive ouverte pluridisciplinaire $\mathbf{H A L}$, est destinée au dépôt et à la diffusion de documents scientifiques de niveau recherche, publiés ou non, émanant des établissements d'enseignement et de recherche français ou étrangers, des laboratoires publics ou privés. 


\title{
Computational determination of the dominant triplet popu- lation mechanism in photoexcited benzophenone
}

\author{
Dumitru-Claudiu Sergentu, ${ }^{a, b}$ Rémi Maurice, ${ }^{a, c}$ Remco W. A. Havenith,,$^{a, d, e}$ Ria Broer ${ }^{a}$ \\ and Daniel Roca-Sanjuán ${ }^{b}$
}

\author{
Received Xth $X X X X X X X X X X$ 20XX, Accepted Xth $X X X X X X X X X$ 20XX \\ First published on the web Xth $X X X X X X X X X X 20 X X$
}

DOI: $10.1039 /$ b000000x

In benzophenone, intersystem crossing occurs efficiently between $\mathrm{S}_{1}\left(n \pi^{*}\right)$ and the $\mathrm{T}_{1}$ state of dominant $n \pi^{*}$ character, leading to excited triplet states after photoexcitation. The transition mechanism between $\mathrm{S}_{1}\left(n \pi^{*}\right)$ and $\mathrm{T}_{1}$ is still a matter of debate, despite several experimental studies. Quantum mechanical calculations have been performed in order to asses the relative efficiencies of previously proposed mechanisms, in particular the direct $\mathrm{S}_{1} \rightarrow \mathrm{T}_{1}$ and indirect $\mathrm{S}_{1} \rightarrow$ $\mathrm{T}_{2}\left(\pi \pi^{*}\right) \rightarrow \mathrm{T}_{1}$ ones. Multiconfigurational wave function based methods are used to discuss the nature of the relevant states and also to determine minimum energy paths and conical intersections. It is found that the $\mathrm{T}_{1}$ state has a mixed $n \pi^{*} / \pi \pi^{*}$ character and that the $\mathrm{T}_{2}\left(\pi \pi^{*}\right)$ state acts as an intermediate state between the $\mathrm{S}_{1}$ and $\mathrm{T}_{1}$ states. This result is in line with recent experiments, which suggested a two-step kinetic model to populate the phosphorescent state after photoexcitation [Aloïse et al., J. Phys. Chem. A 2008, 112, 224-231].

\section{Introduction}

After photoexcitation to the $\mathrm{S}_{1}\left(n \pi^{*}\right)$ state, benzophenone, $\mathrm{C}_{13} \mathrm{H}_{12} \mathrm{O}$, efficiently relaxes radiationless to the $\mathrm{T}_{1}$ excited state, which is thought to have $n \pi^{*}$ character, via a spin-orbit coupling (SOC) driven mechanism. This phenomenon occurs in single-crystals, ${ }^{1}$ in solution ${ }^{2}$, and even in isolated matrices. ${ }^{3}$ Its existence is then independent on the environment, while its efficiency can be affected by it via a change in the tilting angle between the phenyl planes, ${ }^{1,4}$ or via the existence of other (competing) photophysical mechanisms. ${ }^{1}$ The present work focusses on the intersystem crossing (ISC) in the gas phase, and will not deal with other phenomena specific to con-

† Electronic Supplementary Information (ESI) available: [Geometrical parameters of $S_{0}, S_{1}$ and $T_{1}$, computed at various levels of theory]. See DOI: $10.1039 /$ b000000x/

${ }^{a}$ Zernike Institute for Advanced Materials, University of Groningen, Nijenborgh 4, Groningen 9747 AG, The Netherlands

E-mail:r.broer@rug.nl

${ }^{b}$ Instituto de Ciencia Molecular, Universitat de València, P. O. Box 22085, ES-46071 València, Spain

E-mail: daniel.roca@uv.es

${ }^{c}$ SUBATECH, UMR CNRS 6457, IN2P3/EMN Nantes/Université de Nantes, 4 Rue A. Kastler, BP 20722, 44307 Nantes Cedex 3, France

E-mail:remi.maurice@subatech.in2p3.fr

${ }^{d}$ Stratingh Institute for Chemistry, University of Groningen, Nijenborgh 4, 9747 AG Groningen, The Netherlands

${ }^{e}$ Ghent Quantum Chemistry Group, Department of Inorganic and Physical Chemistry, Ghent University, Krijgslaan 281 (S3), B9000 Ghent, Belgium densed states.

According to El-Sayed's rules, ${ }^{5}$ an ISC process governed by direct SOC interaction is fast if the transition involves a change in the molecular orbital type. Hence, assuming almost pure $n \pi^{*}$ characters for both $\mathrm{S}_{1}$ and $\mathrm{T}_{1}$ states, the direct ISC rate constant should be low. ${ }^{4}$ As evidenced by Yabumoto et al. ${ }^{6}{ }^{6} \mathrm{~T}_{2}\left(\pi \pi^{*}\right)$ excited state lies almost isoenergetically with $\mathrm{S}_{1}$, which could allow a fast $\mathrm{S}_{1}\left(n \pi^{*}\right) \rightarrow \mathrm{T}_{2}\left(\pi \pi^{*}\right)$ transition based on the same reasoning. However, if $\mathrm{T}_{1}$ has a mixed $n \pi^{*} / \pi \pi^{*}$ character, the direct $\mathrm{S}_{1} \rightarrow \mathrm{T}_{1}$ transition may become as efficient as the $\mathrm{S}_{1} \rightarrow \mathrm{T}_{2}$ one, and thus knowledge of the actual nature of the electronic states can be particularly helpful to discuss the efficiency of the main potential mechanisms. The nature of the involved electronic states is actually not the only factor affecting the transition rates. In order to discriminate between the two envisaged mechanisms (direct, i.e., $\mathrm{S}_{1} \rightarrow \mathrm{T}_{1}$, or indirect, i.e., $\mathrm{S}_{1} \rightarrow \mathrm{T}_{2} \rightarrow \mathrm{T}_{1}$ ), a computational study based on an accurate determination of the decay channels using the photochemical reaction path approach ${ }^{7-11}$ is particularly promissing. The aim of the present study is therefore to determine, by means of minimum energy path (MEP) computations, the most favorable decay paths of benzophenone to the emissive $\mathrm{T}_{1}$ state.

The paper is structured as follows: a review of the main findings and conclusions obtained in previous experimental studies is first done, then the computational details are discussed followed by a presentation and discussion 
of the results, and finally the conclusions are given.

\section{Survey of previous experimental stud- ies}

In the last decades, the fast ISC that occurs in benzophenone as well as in other related aromatic ketones has been intensively studied using different time-resolved techniques. Since some results may be contradictory, we here review the important aspects of these works in order to prepare the discussion of our computational results.

El-Sayed and Leyerle ${ }^{12}$ used the low field Zeeman effect in order to elucidate a possible indirect $\mathrm{S}_{1} \rightarrow \mathrm{T}_{2} \rightarrow$ $\mathrm{T}_{1}$ mechanism and totally ruled it out. Their results suggest a direct $\mathrm{S}_{1}\left(n \pi^{*}\right) \rightarrow \mathrm{T}_{1}\left(n \pi^{*}\right)$ ISC for benzophenone in bis(p-bromophenyl)ether at $1.6 \mathrm{~K}$. Another work that strongly supports direct ISC between the $\mathrm{S}_{1}$ and $\mathrm{T}_{1}$ excited states was carried out by the group of Ohmori et $a l .{ }^{3}$ In this study, the sensitized phosphorescence excitation spectra of jet-cooled benzophenone was measured in the $\mathrm{S}_{0} \rightarrow \mathrm{S}_{1}$ and $\mathrm{S}_{0} \rightarrow \mathrm{T}$ regions. Following this, an $\mathrm{S}_{1}\left(n \pi^{*}\right)$ minimum was encountered at $26.180 \mathrm{~cm}^{-1}$ $(3.25 \mathrm{eV})$ and another one related to the $\mathrm{T}_{1}\left(n \pi^{*}\right)$ state at $24.224 \mathrm{~cm}^{-1}(3.00 \mathrm{eV})$. The absence of $\mathrm{T}\left(\pi \pi^{*}\right)$ signals in the proximity of $\mathrm{T}_{1}\left(n \pi^{*}\right)$ led to the idea that this state would lie well above $\mathrm{S}_{1}$, in agreement with El-Sayed et $a l .{ }^{12}$ and also other experiments with benzophenone and 4,4'-diiodobenzophenone crystals. ${ }^{13}$

Matushita et al. ${ }^{14}$ studied the photochemical reaction of excited benzophenone in the gas phase. After photoexcitation at $337 \mathrm{~nm}$ of 1.0 Torr benzophenone -10.0 Torr 1,4-cyclohexadiene system, the absorption spectrum is characterized by a weak $\mathrm{S}_{1}\left(n \pi^{*}\right)$ band at $\sim 350 \mathrm{~nm}$ and more intense bands starting at $\sim 310 \mathrm{~nm}$ due to population of levels with $\pi \pi^{*}$ character. The kinetic studies on the decay process of triplet benzophenone revealed longliving emission that follows a biexponential decay. This was attributed to a dual phosphorescence, (i) from an unrelaxed benzophenone triplet state and (ii) from the vibrationally relaxed levels of the triplet state.

Katoh et al. ${ }^{1}$ analyzed the ISC process in a benzophenone single crystal using the picosecond time-resolved absorption spectroscopy technique. The study revealed an ISC rate of $0.04 \pm 0.003 \mathrm{ps}^{-1}$ which is lower than those measured in solution, $0.11 \pm 0.03 \mathrm{ps}^{-1}$ in acetonitrile and $0.06 \pm 0.01 \mathrm{ps}^{-1}$ in isooctane. ${ }^{15}$ Another important feature observed in the benzophenone single crystal was a faster growth of the triplet exciton band compared to the decay of the singlet exciton band. This led to the idea that, besides the population of the triplet exciton through ISC with the lowest singlet exciton, another pro- cess which is more rapid has to be involved. This process was identified as a fission of a polymolecular highly excited state, populated by a biphotonic process, into two triplet excitons.

Yabumoto et al. ${ }^{6}$ measured the transient infrared spectra of a carbon tetrachloride solution of benzophenone in the lowest triplet excited state, using the nanosecond time-resolved infrared spectroscopy technique. The $\mathrm{S}_{1}\left(n \pi^{*}\right)$ state was populated at the $349 \mathrm{~nm}$ photoexcitation. The infrared spectrum of $T_{1}$ in the case of benzene and carbon tetrachloride solution of benzophenone lacked the peak related to the $\mathrm{CO}$ stretch vibration allowing to conclude the $n \pi^{*}$ nature for this state of interest. A band with a broad feature in the wavenumber region of $\sim 2000 \mathrm{~cm}^{-1}$ was identified and assigned to the $\mathrm{T}_{1} \rightarrow \mathrm{T}_{2}$ transition. The results indicate that the $0-0$ energy gap between $T_{1}$ and $T_{2}$ is lower than $2000 \mathrm{~cm}^{-1}$, while the gap between $S_{1}$ and $T_{1}$ is known to be $\sim 1950 \mathrm{~cm}^{-1}$. $^{3}$ Hence, $S_{1}$ and $T_{2}$ must be very close in terms of energy. In this scenario, the El-Sayed rules are respected for the $\mathrm{S}_{1} \rightarrow \mathrm{T}_{2}$ ISC and together with an ultrafast $\mathrm{T}_{2} \rightarrow \mathrm{T}_{1}$ internal conversion (IC), it could confer an efficient indirect $\mathrm{S}_{1} \rightarrow \mathrm{T}_{1}$ conversion.

Aloïse et al. $^{2}$ closely analyzed in 2008 the $\mathrm{S}_{1}\left(n \pi^{*}\right)$ $\rightarrow \mathrm{T}_{1}\left(n \pi^{*}\right)$ ISC by means of ultrafast absorption spectroscopy and multivariate curve resolution, and proposed a two-step kinetic model $\mathrm{S}_{1} \rightarrow$ IS $\rightarrow \mathrm{T}_{1}$ (where IS denotes an intermediate state). With this model, they obtained $\sim 6.5$ and $\sim 10$ ps characteristic times, respectively. The transient absorption spectra of benzophenone in acetonitrile were recorded for two excitation wavelengths, 383 and $267 \mathrm{~nm}$. The peak of the IS of benzophenone in between $\mathrm{S}_{1}$ and $\mathrm{T}_{1}$ was found at $\Delta \lambda_{\max }\left(\mathrm{IS}_{\mathrm{S}} \mathrm{T}_{1}\right)=$ $13 \mathrm{~nm}$ and $\Delta \lambda_{\max }\left(\mathrm{S}_{1}\right.$-IS $)=41 \mathrm{~nm}$ in the case of the $267 \mathrm{~nm}$ excitation, and at $\Delta \lambda_{\max }\left(\mathrm{IS}-\mathrm{T}_{1}\right)=5 \mathrm{~nm}$ and $\Delta \lambda_{\max }\left(\mathrm{S}_{1}\right.$-IS $)=40 \mathrm{~nm}$ in the case of the $383 \mathrm{~nm}$ excitation. The differences between the former values illustrates an excitation wavelength effect: with the 383 $\mathrm{nm}$ excitation, $\mathrm{S}_{1}$ is populated near the $\nu=0$ vibrational level while more vibrational levels are populated with the $267 \mathrm{~nm}$ excitation, through a suggested $\mathrm{S}_{2}\left(\pi \pi^{*}\right)$ $\mathrm{S}_{1}\left(n \pi^{*}\right) \mathrm{IC}$ process. The similarities found between the IS and $T_{1}$ spectra raised the suggestion that the IS species could be related to one or several vibrational levels of the $\mathrm{T}_{1}\left(n \pi^{*}\right)$ manifold, involved in two electronic transitions. The experiment was repeated in different solvents like acetonitrile, methanol, and dichloromethane, and the results confirm that the ISC process is not influenced significantly by the solvent polarity. In contrast, in the substituted molecule 4-methoxybenzophenone the relative position of the lowest two triplet states is switched while the solvent polarity increases, i.e., the lowest triplet state has 
$\pi \pi^{*}$ character instead of $n \pi^{*}$ for polar enough solvents.

In conclusion, whether $\mathrm{T}_{2}\left(\pi \pi^{*}\right)$ is a true spectroscopic state relevant in the photochemistry of benzophenone is still a matter of debate. To the best of our knowledge, no theoretical work has been carried out in order to clearly support one of the mechanisms suggested in the experimental works. In order to elucidate and discuss the efficiency of the main photochemical decay paths for an efficient triplet population in photoexcited benzophenone, we performed quantum-mechanical calculations, as described in the following section.

\section{Computational details}

The ground state $\left(\mathrm{S}_{0}\right)$ geometry of benzophenone was first optimized with density functional theory (DFT) and the def2-TZVP basis set ${ }^{16}$ using the TURBOMOLE program package, version $6.4 .^{17}$ The PBE0 ${ }^{18,19}$ hybrid functional was used. The geometry was also further optimized using the complete active space self-consistent field (CASSCF) 20,21 method and the L-type atomic natural orbitals double- $\zeta$ plus polarization basis set (ANOL-VDZP) ${ }^{22,23}$ with MOLCAS. ${ }^{24}$ MEPs were computed at the same CASSCF/ANO-L-VDZP level of theory by following steepest descents, as described elsewhere. ${ }^{25}$ Mass-weighted coordinates were used. SOCs were computed with the restricted active space state-interaction (RASSI) method ${ }^{26,27}$ using the atomic mean-field approximation. ${ }^{28,29}$ The CASSCF method introduces static electron correlation and is known to be able to produce a qualitatively correct description of multiconfigurational states, provided that the active space is well chosen. Dynamic correlation is then introduced with the complete active space second-order perturbation theory (CASPT2) method ${ }^{30,31}$ at the CASSCF optimized geometries (CASPT2//CASSCF). In order to prevent the occurence of intruder states, an imaginary level-shift ${ }^{32}$ of 0.2 a.u., was used. The ionization potential electron affinity (IPEA) shift ${ }^{33}$ was set to 0.00 in all the reported calculations.

The relevant orbitals for characterizing the lowest-lying excited states of the $\left(\mathrm{C}_{6} \mathrm{H}_{5}\right)_{2} \mathrm{CO}$ benzophenone molecule are the valence $\pi$ and $\pi^{*}$ orbitals plus the $n$ orbital (i.e., the lone pair) of the oxygen atom, which corresponds to 16 electrons and 15 orbitals. In the present study, two pairs of correlated $\pi$ and $\pi^{*}$ orbitals, which would have the largest and smallest occupation numbers in a CASSCF(16/15) calculation, were not included in the active space. Hence, geometry optimizations of the states of interest and MEPs were carried out at the CASSCF(12/11) level of theory.

Test calculations were performed at the Franck-
Condon (FC) region to evaluate the accuracy and convergence of the CASPT2/ANO-LVDZP//CASSCF(12/11)/ANO-L-VDZP results with respect to the size of the active space and the size of the basis set. Indeed, the vertical transition energies $\left(\Delta E_{\mathrm{V}}\right)$ at the CASSCF $(12 / 11) /$ ANO-L-VDZP optimized ground-state geometry were also computed with the valence triple- $\zeta$ quality ANO-L-VTZP basis set, while a larger active space, including all the valence $\pi$ system, was also considered with the valence double- $\zeta$ basis. Due to its high associated computational cost, the test calculation with the largest active space was carried out imposing a $C_{2}$ symmetry. In addition, transition energies were computed with CASPT2 at the PBE0 $\mathrm{S}_{0}$ optimized geometry and compared with the corresponding energies obtained with CASPT2//CASSCF.

Calculations were also performed with the restrictedactive-space second-order perturbation theory (RASPT2) method. ${ }^{34,35}$ In this approach, the active space is divided into three different subspaces: RAS1, RAS2, and RAS3. From the orbitals included in the RAS2 subspace, all possible configuration state functions (CSFs) of a particular spin and spatial symmetry are included in the expression of the multiconfigurational wave functions. The orbitals included in the RAS1 and RAS3 subspaces allow the generation of some aditional CSFs that are subject to restrictions: the maximum number of holes allowed in RAS1 and the maximum number of particles allowed in RAS3 are defined by the user. The $\pi$ and $\pi^{*}$ orbitals and the oxygen lone pair can be distributed in the different subspaces in various ways. The $\operatorname{RASSCF}(2,11,2), \operatorname{RASSCF}(4,7,4), \operatorname{RASSCF}(6,3,6)$ reference wave functions were used, where the numbers in parenthesis refer to the number of orbitals in the RAS1, RAS2, and RAS3 subspaces, respectively. A maximum of 2 holes is allowed in RAS1 and a maximum of 2 particles in RAS3. Furthermore, the transition energies were computed with time-dependent DFT with the PBE0 exchange-correlation functional (TD-PBE0) and equation of motion coupled cluster singles and doubles (EOM-CCSD) methods with the def2-TZVP and cc-pVDZ basis sets, respectively. The TD-DFT calculations have been performed with NWChem ${ }^{36}$ (release 6.1.1), while the CCSD ones have been performed with Dalton (release 2011). ${ }^{37}$

Four singlet and four triplet states were averaged in separate singlet and triplet $\operatorname{CASSCF}(12 / 11)$ calculations, respectively. When the ground state DFT geometry is used, the averaging scheme over four triplet states did not lead to convergence on a similar active space as the one obtained at the CASSCF optimized geometry. Therefore, the triplet CASSCF refer- 
ence wave functions in the CASPT2//DFT calculations were built with a different state-averaging scheme than the CASPT2//CASSCF ones, i.e five triplet states were considered. Regarding the CASPT2(16/15)/ANO-LVDZP//CASSCF(12/11)/ANO-L-VDZP computations with the $C_{2}$ symmetry constraint, four singlet and four triplet states were separately averaged in each irreducible representation, leading to four sets of orbitals and states.

\section{Results and discussions}

The presentation and discussion of the results is split in two parts. First, a comparative analysis of the relevant geometries for decay mechanisms of interest is provided. Then energies and natures of the lowest-lying singlet and triplet states at the ground state geometry are discussed. The main decay channels of benzophenone are finally described in a second part.

\subsection{Ground state geometry, Franck-Condon Re- gion and Lowest-Lying Excited State Ge- ometries}

The relevant PBE0/def2-TZVP and the CASSCF(12/11)/ANO-L-VDZP optimized geometry parameters of $\mathrm{S}_{0}, \mathrm{~S}_{1}$ and $\mathrm{T}_{1}$ are compiled in Table $\mathrm{S} 1$, and the geometries are displayed in Figure 1. These results can be compared with the computational data of Sett et al. ${ }^{38}$

A planar geometry for benzophenone could be envisaged assuming no steric repulsion between the phenyl rings. However, a steric repulsion exists between the adjacent hydrogen atoms that belong to two different phenyl rings, preventing the coplanarity of the phenyl groups. The PBE0 hybrid functional predicts a rotation of each phenyl ring with $28^{\circ}$ out of the plane that includes the $\mathrm{CO}$ bond and bissects the two phenyl planes. A similar rotation angle $\left(31^{\circ}\right)$ is predicted at the CASSCF(12/11)/ANO-L-VDZP level. The values are in a good agreement with previously reported theoretical ones. ${ }^{38,39}$ Note that our results are also in line with the crystal structure reported by Fleischer et al. in 1968, ${ }^{40}$ indicating only a moderate effect of the environment on the ground state geometry of benzophenone.

Significant geometrical changes are expected for the excited states, which is confirmed by the present calculations, as can be seen in Table S1 and Figure 1. The $\mathrm{CO}$ bond distance in the $\mathrm{S}_{0}$ geometry is computed to be $1.21 \AA$ with $\operatorname{CASSCF}(12 / 11)$, which is in agreement with the DFT results. It is elongated in the excited $\mathrm{S}_{1}$ and $\mathrm{T}_{1}$ states for which the CASSCF values are 1.37 and $1.35 \AA$, respectively. These results are consistent with the fact that the electronic structure of the $S_{1}$ and $T_{1}$ states is characterized by an electron promotion from a non-bonding $n$ orbital to an orbital with $\mathrm{CO} \pi^{*}$ character. Also, the angle between the phenyl planes is reduced from $58^{\circ}$ in the $\mathrm{S}_{0}$ optimized geometry to 40 and $44^{\circ}$ in the $\mathrm{S}_{1}$ and $\mathrm{T}_{1}$ geometries, respectively, at the $\operatorname{CASSCF}(12 / 11)$ level. This suggests electron delocalization over the two phenyl groups in the $\mathrm{S}_{1}$ and $\mathrm{T}_{1}$ excited states.

As mentioned above, the optimal active space for this system would correspond to 15 active orbitals, including $6 \pi$ and $\pi^{*}$ orbitals located on each phenyl ring, the $\pi$ and $\pi^{*}$ on the CO double bond, and the lone pair $\left(n_{\mathrm{O}}\right)$ orbital containing the two non-bonding electrons of the oxygen atom (see Figure 2). As the number of CSFs increases with the number of active orbitals comprising the active space in the CASSCF formalism, calculations using the total amount of 16 electrons and 15 orbitals become computationally too demanding for performing CASSCF geometry optimizations. In order to find an affordable approach with enough accuracy for the aim of the present work, high-level CASPT2(16/15) computations were performed at the FC geometry and the results were compared with the values obtained with less demanding approaches including the CASPT2(12/11) one and various RASPT2 ones. Taking into account the symmetry properties of the $\mathrm{S}_{0}$ equilibrium structure and in order to both reduce the computational cost and get insight on more excited states, $C_{2}$ (orbital) symmetry was imposed in the CASPT2(16/15) calculations. Averaging the CASSCF orbitals between several states while imposing $C_{2}$ symmetry could lead to different results than a state-average performed in the $C_{1}$ symmetry point group. Also, note that MOLCAS only averages orbitals between states of the same spin multiplicity and spatial symmetry. In order to find a good combination of active space and averaging scheme, both $C_{1}$ and $C_{2}$ point groups are considered and the obtained results compared. The highlevel CASPT2(16/15) calculations are taken as a reference to assess the validity of the CASPT2(12/11) ones.

Table 1 compiles the CASPT2(16/15)//CASSCF(12/11) vertical excitation energies $\Delta E_{\mathrm{V}}$ and oscillator strengths $f$. The lowest-energy electronic state leading to the most intense absorption is located at $5.39 \mathrm{eV}(\sim 230 \mathrm{~nm})$ and corresponds to the $\mathrm{S}_{0} \rightarrow \mathrm{S}_{4}\left(\pi \pi^{*}\right)$ transition. The $\mathrm{S}_{1}$ excited state lies at $3.66 \mathrm{eV}(\sim 338 \mathrm{~nm})$. The $n \pi^{*}$ character of this state corresponds mainly to an excitation from the oxygen lone pair $\left(n_{\mathrm{O}}\right.$ in Figure 2$)$ to the anti-bonding $\pi^{*}$ orbital of the carbonyl ( $\Pi_{1}^{*}$ in Figure 2). There are three triplet states identified in the proximity of $\mathrm{S}_{1}$, located vertically at $3.33 \mathrm{eV}(\sim 372 \mathrm{~nm}), 3.41 \mathrm{eV}(\sim 362$ $\mathrm{nm})$, and $3.69 \mathrm{eV}(\sim 335 \mathrm{~nm})$ above the ground state. 

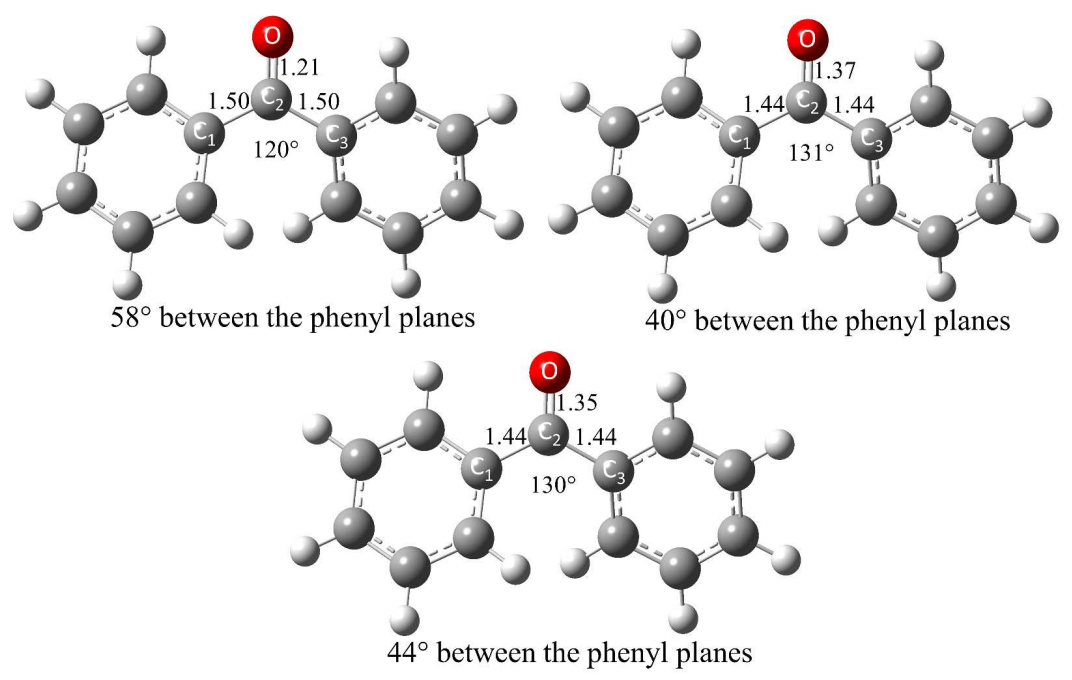

Fig. 1 CASSCF(12/11)/ANO-L-VDZP optimized geometries of the $\mathrm{S}_{0}$ (top left), $\mathrm{S}_{1}$ (top right), and $\mathrm{T}_{1}$ (bottom) states. Bond lengths are given in ångström. Atom labels are displayed.

Table 1 Vertical excitation energies computed at the CASPT2(16/15)/ANO-L-VDZP//CASSCF(12/11)/ANO-L-VDZP level of theory, and oscillator strengths and dipole moments of the corresponding reference states $\left(C_{2}\right.$ point group symmetry).

\begin{tabular}{llllllll}
\hline \multicolumn{2}{c}{ A symmetry } & \multicolumn{5}{c}{ B symmetry } \\
\hline State & $\Delta E_{\mathrm{V}}(\mathrm{eV})$ & $f$ & $\mu(\mathrm{D})$ & State & $\Delta E_{\mathrm{V}}(\mathrm{eV})$ & $f$ & $\mu(\mathrm{D})$ \\
\hline $\mathrm{S}_{0}$ & 0 & & 2.76 & & & & \\
$\mathrm{~S}_{1}\left(n \pi^{*}\right)$ & 3.66 & 0.001 & 0.96 & & & & \\
$\mathrm{~S}_{2}\left(\pi \pi^{*}\right)$ & 4.33 & 0.003 & 2.94 & & & & \\
& & & & $\mathrm{~S}_{3}\left(\pi \pi^{*}\right)$ & 4.43 & 0.001 & 2.92 \\
& & & & $\mathrm{~S}_{4}\left(\pi \pi^{*}\right)$ & 5.39 & 0.150 & 3.52 \\
$\mathrm{~T}_{1}\left(n \pi^{*} / \pi \pi^{*}\right)$ & 3.33 & 0.000 & 2.47 & & & & \\
$\mathrm{~T}_{2}\left(\pi \pi^{*}\right)$ & 3.41 & 0.000 & 1.09 & & & & \\
& & & & $\mathrm{~T}_{3}\left(\pi \pi^{*}\right)$ & 3.69 & 0.000 & 2.95 \\
& & & & $\mathrm{~T}_{4}\left(\pi \pi^{*}\right)$ & 4.18 & 0.000 & 2.95 \\
$\mathrm{~T}_{5}\left(\pi \pi^{*}\right)$ & 4.18 & 0.000 & 2.70 & & & & \\
$\mathrm{~T}_{6}\left(\pi \pi^{*}\right)$ & 4.22 & 0.000 & 2.90 & & & & \\
& & & & $\mathrm{~T}_{7}\left(\pi \pi^{*}\right)$ & 4.26 & 0.000 & 2.92 \\
& & & & $\mathrm{~T}_{8}\left(\pi \pi^{*}\right)$ & 6.47 & 0.000 & 2.77 \\
\hline
\end{tabular}

This journal is @ The Royal Society of Chemistry [year] 


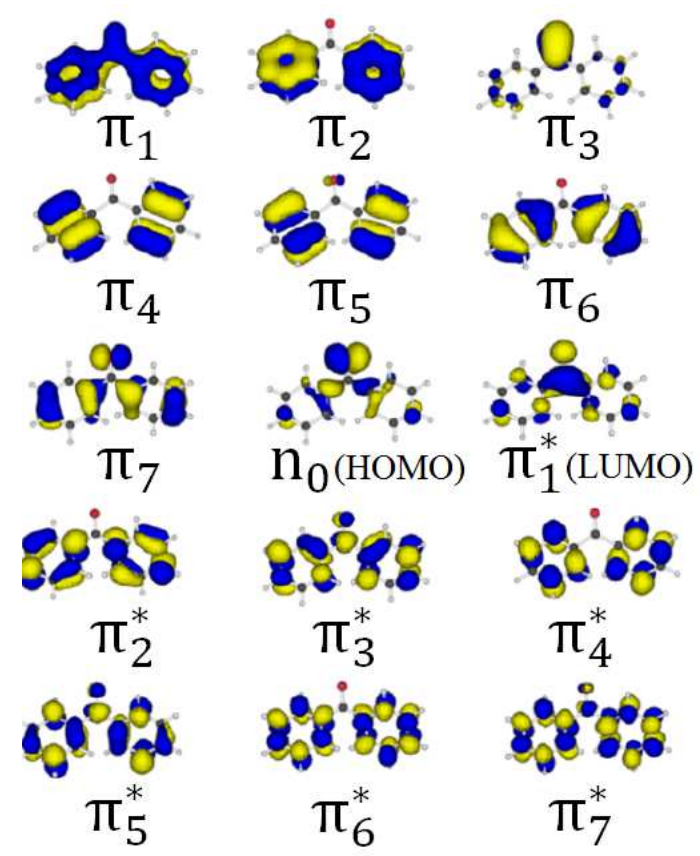

Fig. 2 SA-CASSCF active space natural orbitals of benzophenone. The natural orbitals that are similar to the highest-occupied molecular orbital (HOMO) and lowest-unoccupied molecular orbital (LUMO) of a ground-state, single-configuration SCF calculation are specifically labeled.
The knowledge of the nature of these states is crucial to understand the relaxation mechanisms of photoexcited benzophenone. On the basis of the present calculations, the lowest-lying triplet state $\mathrm{T}_{1}$ has a mixed $n \pi^{*} / \pi \pi^{*}$ character and it is followed closely $(0.08 \mathrm{eV})$ by a state of pure $\pi \pi^{*}$ character, namely $\mathrm{T}_{2}$. Note that these characters have been obtained by looking at the dominant configurations in the corresponding multiconfigurational wave functions, and that the orbitals have been labeled according to their dominant characters, as can be seen in Figure 2. Note that such labeling is somehow arbitrary, since no symmetry element strictly separates the $n$, $\pi$ and $\pi^{*}$ type orbitals in benzophenone. The dipole moments $\mu$ computed with $\operatorname{CASSCF}(16 / 15)$ are 2.76, 0.96 , and $2.47 \mathrm{D}$ for $\mathrm{S}_{0}, \mathrm{~S}_{1}$, and $\mathrm{T}_{1}$, respectively, which is qualitatively in line with the experimental values of $2.98,1.23$, and $1.72 \mathrm{D}$, respectively, measured in benzophenone crystals. ${ }^{41,42}$ Dipole moments allow to roughly analyze the influence of different solvents. It is estimated that a polar solvent may induce a blueshift of the $\mathrm{S}_{1}\left(n \pi^{*}\right)$ band and a redshift of the other singlet $\pi \pi^{*}$ bands. Concerning triplets, $\mathrm{T}_{1}$ is expected to be stabilized in the same manner as the ground state, while $\mathrm{T}_{2}$ may suffer a slight blueshift. These estimations are in agreement with previous experimental results and other theoretical works on the absorption spectrum. ${ }^{2,43}$ Taking into account these results, the population of the lowest-lying $\mathrm{T}_{1}$ state from the $\mathrm{S}_{1}\left(n \pi^{*}\right)$ state through ISC would be allowed according to El-Sayed rules, since $\mathrm{T}_{1}$ is found to have a mixed $n \pi^{*} / \pi \pi^{*}$ character.

Table 2 shows a comparison between the $\Delta E_{\mathrm{V}}$ values of several states of interest and their main corresponding excitation nature, computed at various levels of theory. Regarding the different CASPT2//CASSCF calculations, the largest discrepancies appear in the $\pi \pi^{*}$ states, which can be attributed to a deficiency of CASPT2(12/11), in comparison to CASPT2(16/15), to accurately describe the excited states of $\pi \pi^{*}$ nature. It is worth reminding at this point that the less relevant correlating pairs of $\pi$ and $\pi^{*}$ orbitals were excluded from the active space in the CASPT2(12/11) calculations. Hence, the $\mathrm{S}_{2}$ state (of $\pi \pi^{*}$ nature) may not be perfectly described, and actually appears at higher energies than when the large active space is used. On the other hand, the $\mathrm{S}_{1}\left(n \pi^{*}\right)$ excited state is accurately described with all the CASPT2//CASSCF approaches. A comparison with the large active space calculation shows a small difference of $0.01 \mathrm{eV}$ when the $C_{2}$ symmetry is imposed and $0.02 \mathrm{eV}$ when the symmetry is not considered. Regarding the $\Delta E_{\mathrm{V}}$ excitation energies of the lowest-lying triplet, the close-lying $\mathrm{T}_{2}$ and $\mathrm{T}_{3}$ states appear in different irreducible representations at the CASPT2(12/11) 
Table 2 Excitation energies computed with several methodologies at the CASSCF(12/11)/ANO-L-VDZP S equilibrium structure. The CASPT2(16/15) excitation energies are reproduced for convenience from Table 1. The ANO-L-VDZP basis set is used for the CASPT2 calculations.

\begin{tabular}{lllll}
\hline & & CASPT2 $(12 / 11)$ & & CASPT2(16/15) \\
\hline & A symmetry & B symmetry & No symmetry & A or B symmetry \\
\hline State & $\Delta E_{\mathrm{V}}(\mathrm{eV})$ & $\Delta E_{\mathrm{V}}(\mathrm{eV})$ & $\Delta E_{\mathrm{V}}(\mathrm{eV})$ & $\Delta E_{\mathrm{V}}(\mathrm{eV})$ \\
\hline $\mathrm{S}_{0}$ & 0.00 & & 0.00 & 0.00 \\
$\mathrm{~S}_{1}(n \pi *)$ & 3.65 & 4.71 & 3.64 & $3.66\left(n \pi^{*}\right)$ \\
$\mathrm{S}_{2}(\pi \pi *)$ & & & 4.67 & $4.33\left(\pi \pi^{*}\right)$ \\
$\mathrm{T}_{1}\left(n \pi^{*} / \pi \pi^{*}\right)$ & 3.25 & 3.43 & 3.11 & $3.33\left(n \pi^{*} / \pi \pi^{*}\right)$ \\
$\mathrm{T}_{2}\left(\pi \pi^{*}\right)$ & & & 3.57 & $3.41\left(\pi \pi^{*}\right)$ \\
$\mathrm{T}_{3}\left(\pi \pi^{*}\right)$ & 3.61 & EOM-CCSD/cc-pVDZ & \\
\hline & TD-PBE0/def2-TZVP & & \\
\hline $\mathrm{S}_{1}\left(n \pi^{*}\right)$ & 3.66 & 4.00 & & \\
$\mathrm{~S}_{2}\left(\pi \pi^{*}\right)$ & 4.74 & 5.06 & \\
$\mathrm{~T}_{1}\left(n \pi^{*} / \pi \pi^{*}\right)$ & 2.98 & 3.49 & \\
$\mathrm{~T}_{2}\left(\pi \pi^{*}\right)$ & 3.41 & 3.88 & & \\
$\mathrm{~T}_{3}\left(\pi \pi^{*}\right)$ & 3.42 & 3.91 & & \\
\hline
\end{tabular}

and CASPT2(16/15) levels when the $C_{2}$ symmetry is imposed. On the other hand, the CASPT2(12/11) approach with the $C_{1}$ point group symmetry performs more accurately as compared to the reference CASPT2(16/15) results. The differences obtained in this case are not larger than $0.2 \mathrm{eV}$ for $\mathrm{T}_{1}, \mathrm{~T}_{2}$ and $\mathrm{T}_{3}$.

The results obtained with TD-DFT and the def2TZVP basis set do not show large discrepancies with the reference CASPT2(16/15) values. Note that the abovementioned CASPT2 and TD-DFT values for the $\mathrm{S}_{0} \rightarrow$ $\mathrm{S}_{1}$ excitation energy are also close to those measured in solution with various solvents ${ }^{44}$ or in crystals ${ }^{45}$ (around $3.6 \mathrm{eV}$ in both the cases). The CCSD/cc-pVDZ level, however, overestimates this transition energy. This may be due to two factors, (i) the double- $\zeta$ Dunning basis set is too small, and (ii) excitations that are not introduced at the CCSD level of theory are important for the excitation energies. Test calculations with a larger (triple zeta) basis set showed similar results for the vertical excitation energies as when the double zeta quality basis set was used, thus issue (ii) is most probably the main one.

Further calculations were carried out in order to test the basis set convergence. The valence triple- $\zeta$ ANO-LVTZP basis set results are thus compared with previously obtained ANO-L-VDZP ones (see Table 3). From the comparison of both sets of values, it can be seen that the triple- $\zeta$ quality basis set does not lead to significant changes as compared to the corresponding double- $\zeta$ basis set, i.e., basis set convergence is nearly achieved, and a double- $\zeta$ basis set can be safely used for more extensive explorations of potential energy surfaces.

It is known that CASSCF optimized geometries may suffer from lack of dynamic electron correlation. Therefore, we also computed the $\Delta E_{\mathrm{V}}$ with the same method
Table 3 Vertical excitation energies computed with the CASPT2(12/11) method and two ANO-L-type basis sets at the $\mathrm{S}_{0}$ equilibrium structure optimized at the CASSCF(12/11)/ANO-L-VDZP level.

\begin{tabular}{lll}
\hline & ANO-L-VDZP & ANO-L-VTZP \\
\hline State & $\Delta E_{\mathrm{V}}(\mathrm{eV})$ & $\Delta E_{\mathrm{V}}(\mathrm{eV})$ \\
\hline $\mathrm{S}_{1}$ & 3.64 & 3.54 \\
$\mathrm{~S}_{2}$ & 4.67 & 4.63 \\
$\mathrm{~S}_{3}$ & 4.67 & 4.66 \\
$\mathrm{~T}_{1}$ & 3.10 & 3.05 \\
$\mathrm{~T}_{2}$ & 3.56 & 3.51 \\
$\mathrm{~T}_{3}$ & 3.59 & 3.56 \\
\hline
\end{tabular}

but using the ground state equilibrium geometry obtained at the DFT(PBE0)/def2-TZVP level of theory (see Table 4). Results in good agreement with the ones obtained with the CASSCF ground state geometry (see Table 2) are found. One should also note that a mixed $n \pi^{*} / \pi \pi^{*}$ character is also obtained for $\mathrm{T}_{1}$, in agreement with the CASPT2//CASSCF results discussed above.

Table 4 CASPT2(12/11)/ANO-L-VDZP vertical excitation energies computed at the $\mathrm{S}_{0} \mathrm{PBE} 0 /$ def2-TZVP optimized structure.

\begin{tabular}{lll}
\hline State & A symmetry & B symmetry \\
\hline & $\Delta E_{\mathrm{V}}(\mathrm{eV})$ & $\Delta E_{\mathrm{V}}(\mathrm{eV})$ \\
\hline $\mathrm{S}_{1}\left(n \pi^{*}\right)$ & 3.58 & \\
$\mathrm{~S}_{2}\left(\pi \pi^{*}\right)$ & 4.59 & \\
$\mathrm{~T}_{1}\left(n \pi^{*} / \pi \pi^{*}\right)$ & 3.20 & \\
$\mathrm{~T}_{2}\left(\pi \pi^{*}\right)$ & & 3.31 \\
$\mathrm{~T}_{3}\left(\pi \pi^{*}\right)$ & 3.54 & \\
\hline
\end{tabular}


From the analysis of the results obtained at different CASPT2//CASSCF levels, we have concluded above that a CAS space comprising only 11 orbitals is not enough to correctly describe the $\mathrm{S}_{2}\left(\pi \pi^{*}\right)$ state. An accurate characterization of the potential energy surface (PES) related to $\mathrm{S}_{2}$ would be important to interpret those experimental works in which the evolution after photoexcitation to this excited state $\left(\mathrm{S}_{2}\right)$ is studied instead or in addition to the analysis of the decay signals after direct population of the $\mathrm{S}_{1}$ state. ${ }^{2,46} \mathrm{In}$ an attempt to improve the description, we followed the possibility of employing the RASSCF/RASPT2 method with the $\operatorname{RAS}(2,11,2), \operatorname{RAS}(4,7,4)$, and $\operatorname{RAS}(6,3,6)$ subspaces (see Table 5). The RASPT2 $(2,11,2)$ level yields results in a very good agreement with the CASPT2(16/15) method. The RAS space is built in such a way that the two $\pi$ orbitals with the occupation numbers closer to two from the CASSCF (16/15) computations are moved to the RAS1 subspace and their correlating counterparts, which are almost unoccupied, are placed in RAS3. The main disadvantage of this method is that it does not significantly reduce the computational cost. Thus, one can try to reduce the RAS2 subspace to only seven orbitals. The resulting RASPT2 $(4,7,4)$ calculations yield results which follow the same trends as the CASPT2(16/15) ones. A further reduction of the RAS2 subspace to only three orbitals leads to very poor results. This issue cannot be fixed by increasing the number of holes in the RAS1 and electrons in the RAS3 space from two to four, due to convergence problems. As already discussed, it is clear that using the $\operatorname{CAS}(12 / 11)$ space within the CASPT2//CASSCF formalism does not lead to a satisfactory description of the $\mathrm{S}_{2}$ state. The $(4,7,4)$ active space within the RASPT2//RASSCF formalism yields some significant improvements, but at a higher computational cost. Hence, the CASPT2(12/11)/ANO-LVDZP//CASSCF(12/11)/ANO-L-VDZP approach will be employed in the characterization of the decay paths mainly focusing on the relaxation from $S_{1}$, as most of the experimental works, while an approximate description will be provided for the decay route after photoexcitation to the $\mathrm{S}_{2}$ state.

Table 5 RASPT2 excitation energies (in eV) computed at the $\mathrm{S}_{0}$ CASSCF(12/11)/ANO-L-VDZP equilibrium structure.

\begin{tabular}{llllll}
\hline Method & $\mathrm{S}_{1}$ & $\mathrm{~S}_{2}$ & $\mathrm{~T}_{1}$ & $\mathrm{~T}_{2}$ & $\mathrm{~T}_{3}$ \\
\hline CASPT2(16/15) & 3.66 & 4.33 & 3.33 & 3.41 & 3.69 \\
RASPT2(2,11,2) & 3.66 & 4.32 & 3.32 & 3.42 & 3.68 \\
RASPT2(4,7,4) & 3.59 & 4.48 & 3.40 & 3.52 & 3.75 \\
RASPT2(6,3,6) & 3.71 & 5.43 & 3.80 & 4.36 & 4.44 \\
\hline
\end{tabular}

\subsection{Energy decay mechanisms}

The main decay paths after irradiation of benzophenone are presented and discussed here in two sub-sections. First, the evolution from the FC region along the lowestlying singlet $\pi \pi^{*}$ and $n \pi^{*}$ excited states is described. Next, the subsequent decay of the triplet states is analyzed.

4.2.1 Singlet manifold. After irradiation with high-energy UV light, the $\mathrm{S}_{4}\left(\pi \pi^{*}\right)$ state, which is the third ${ }^{1}\left(\pi \pi^{*}\right)$ excited state placed at $5.39 \mathrm{eV}$ at the CASPT2(16/15) level (see Table 1), has the largest probability to be populated among the excited states computed in the present work. It is worth noting that a total amount of 7 triplet states of both $n \pi^{*}$ and $\pi \pi^{*}$ nature exist at lower energies than that of $\mathrm{S}_{4}$. Therefore, many singlet-triplet crossings (STCs) can be expected along the decay of the brighest state $\mathrm{S}_{4}$, which will increase the probability for population transfer to the triplet manifold. Nevertheless, the interesting electronic states of benzophenone are those appearing at lower energies (see, for example, ref 47 and references therein). Experimental works to compare with use photoexcitation wavelengths to populate $\mathrm{S}_{2}\left(\pi \pi^{*}\right)$ and especially the lowestlying $\mathrm{S}_{1}\left(n \pi^{*}\right)$ state. At the CASPT2(12/11)/ANO-LVDZP//CASSCF(12/11)/ANO-L-VDZP level, the $\Delta E_{\mathrm{V}}$ transition energies to $\mathrm{S}_{1}$ and $\mathrm{S}_{2}$ are 3.64 and $4.67 \mathrm{eV}$, respectively, and the associated oscillator strengths $f$ values are 0.001 and 0.008 , respectively. Thus, the $f$ related to the ${ }^{1}(\pi \pi)^{*}$ state is one order of magnitude higher than that related to the ${ }^{1}(n \pi)^{*}$ state. This behavior can be understood more clearly in a planar $C_{2 \mathrm{v}}$ molecule. In such a case, the $\mathrm{S}_{0} \rightarrow^{1}(\pi \pi)^{*}$ transition is generally allowed, while the $\mathrm{S}_{0} \rightarrow{ }^{1}(n \pi)^{*}$ transition is not. Since, in the non-planar case, $\mathrm{S}_{1}$ has a mixed $n \pi^{*} / \pi \pi^{*}$ character, this transition is allowed, and the transition probability is related to the $n \pi^{*} / \pi \pi^{*}$ mixing, such that:

$$
f\left(S_{0} \rightarrow n \pi^{*} / \pi \pi^{*}\right)=\left|\frac{\left\langle n \pi^{*}|H| \pi \pi^{*}\right\rangle}{E_{\pi \pi^{*}}-E_{n \pi^{*}}}\right|^{2} f\left(S_{0} \rightarrow \pi \pi^{*}\right)
$$

where the notations $n \pi^{*}$ and $\pi \pi^{*}$ correspond, according to perturbation theory, to the zeroth-order states and the Hamiltonian $H$ includes the (electronic) interactions that mix these two zeroth-order states. The first term in the right-hand side of Eq. 1 is defined as the mixing coefficient, $\lambda$. It follows that the magnitude of $f$ for the $\mathrm{S}_{0} \rightarrow \mathrm{S}_{1}\left(n \pi^{*}\right)$ transition depends on the magnitude of the electronic matrix element between the zeroth-order $\left(n \pi^{*}\right)$ and $\left(\pi \pi^{*}\right)$ states, their energy gap, and the zerothorder $f$ for the $\mathrm{S}_{0} \rightarrow \pi \pi^{*}$ transition. Note that since the $n \pi^{*} / \pi \pi^{*}$ mixing of $\mathrm{S}_{1}$ is weak (it is almost pure $n \pi^{*}$ in 


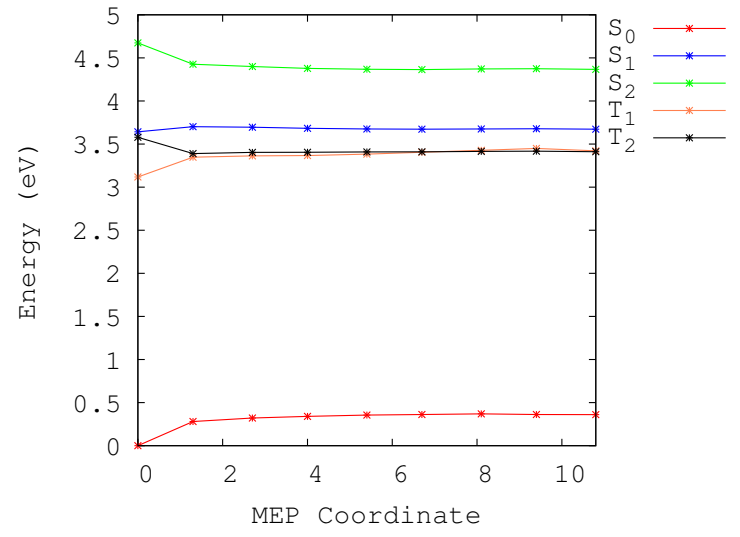

Fig. 3 CASPT2(12/11)//CASSCF(12/11) energies of the ground and lowest-lying singlet and triplet excited states of benzophenone along the MEP on the PES of the $\mathrm{S}_{2}\left(\pi \pi^{*}\right)$ state from the FC region toward $\mathrm{S}_{2}\left(\pi \pi^{*}\right)_{\text {min }}$.

the $\mathrm{FC}$ region), we will refer to this state in the remainder of the text as $\mathrm{S}_{1}\left(n \pi^{*}\right)$.

The initial evolution of the system after light irradiation and population of the low-energy $\mathrm{S}_{2}\left(\pi \pi^{*}\right)$ state is shown in Figure 3. $\mathrm{S}_{2}\left(\pi \pi^{*}\right)$ evolves directly toward its relaxed non-planar minimum, $\mathrm{S}_{2}\left(\pi \pi^{*}\right)_{\text {min }}$, placed adiabatically (i.e., with respect to the energy of the $\mathrm{S}_{0}$ state at its optimized geometry) at $4.36 \mathrm{eV}$. The geometry of the $\mathrm{S}_{2}\left(\pi \pi^{*}\right)$ minimum (Figure 4) is characterized by a slight elongation of the CO bond (1.23 $\AA$ ), coplanarity of one phenyl ring with the carbonyl group, and an angle of $51^{\circ}$ between the phenyl rings. In Figure 3, it can be seen that the $\mathrm{S}_{2}$ MEP does not lead to a $\mathrm{S}_{2}\left(\pi \pi^{*}\right) / \mathrm{S}_{1}\left(n \pi^{*}\right)$ conical intersection (CI). The energy splitting, at the end of the MEP, between these two states is still large, $0.69 \mathrm{eV}$. Taking into account that the CASPT2(12/11)//CASSCF(12/11) level used in the present study leads to a too high $\mathrm{S}_{2}$ relative energy (as concluded in section 4.1), a barrierless or almost barrierless decay path towards a $\mathrm{S}_{2} / \mathrm{S}_{1}$ CI cannot be discarded. Recent experimental works studying the decay mechanisms after photoexcitation to $\mathrm{S}_{2}$ supported a fast IC process for population transfer to the lowest-lying $\mathrm{S}_{1}$ excited state. ${ }^{46}$

Next, we focus on the relaxation from the $\mathrm{S}_{1}\left(n \pi^{*}\right)$ state that can be for instance reached after photoexcitation to $\mathrm{S}_{2}$ or direct photoexcitation. Considering the latter case, the evolution on the PES of the $\mathrm{S}_{1}\left(n \pi^{*}\right)$ excited state, starting at the $\mathrm{FC}$ region is shown in Figure 5. Note that the MEP on the $\mathrm{S}_{1}$ surface evolves directly to the equilibrium structure of the state, $\mathrm{S}_{1}\left(n \pi^{*}\right)_{\min }$, located adia-

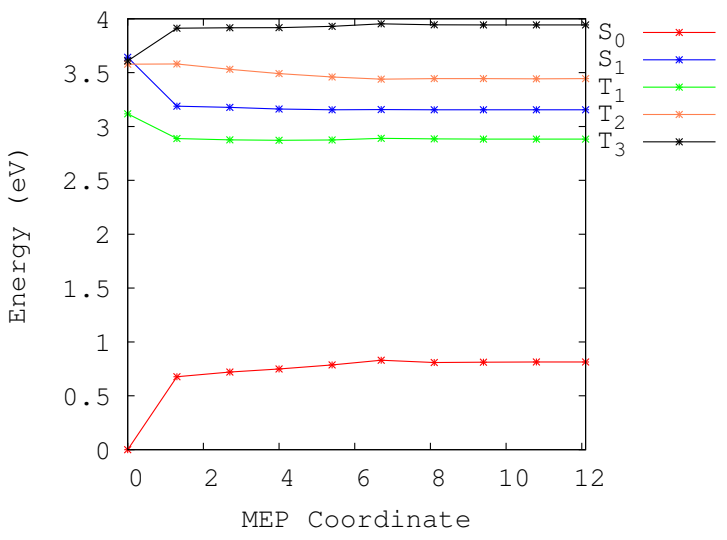

Fig. 5 CASPT2(12/11)//CASSCF(12/11) energies of the ground and lowest-lying singlet and triplet excited states of benzophenone along the MEP on the PES of the $\mathrm{S}_{1}\left(n \pi^{*}\right)$ state from the $\mathrm{FC}$ region toward $\mathrm{S}_{1}\left(n \pi^{*}\right)_{\min }$.

batically at $3.15 \mathrm{eV}$ above $\mathrm{S}_{0}$ (the experimental 0-0 energy difference being $3.25 \mathrm{eV}) .{ }^{3}$ Along the MEP reported for $\mathrm{S}_{1}\left(n \pi^{*}\right)$, four different decay mechanisms, which are anticipated here and described in detail below, may be active:

- $\mathrm{S}_{1}(n \pi)^{*} / \mathrm{T}_{3}\left(\pi \pi^{*}\right)$ ISC followed by IC from $\mathrm{T}_{3}$ to $\mathrm{T}_{2}$, IC from $\mathrm{T}_{2}$ to $\mathrm{T}_{1}$ and finally phosphorescence from $\mathrm{T}_{1}$ to $\mathrm{S}_{0}$.

- $\mathrm{S}_{1}(n \pi)^{*} / \mathrm{T}_{2}\left(\pi \pi^{*}\right)$ ISC followed by IC and then phosphorescence from $T_{1}$ to $S_{0}$.

- direct $\mathrm{S}_{1}(n \pi)^{*} / \mathrm{T}_{1}$ ISC, and phosphorescence from $\mathrm{T}_{1}$ to $\mathrm{S}_{0}$.

- fluorescence from $\mathrm{S}_{1}$ to $\mathrm{S}_{0}$.

In order to estimate the probability for singlet-triplet population transfer from $S_{1}$ to $T_{2}$ and $T_{3}$, the $S O C$ matrix elements between the spin components of $\mathrm{S}_{1}$ and $T_{1}, S_{1}$ and $T_{2}$, and $S_{1}$ and $T_{3}$ are determined in the $\mathrm{FC}$ region, using the spin-orbit RASSI (SO-RASSI) method. ${ }^{26,27}$ SOC magnitudes of 24 and $22 \mathrm{~cm}^{-1}$ are obtained between the $\mathrm{S}_{1}\left(n \pi^{*}\right)$ and $\mathrm{T}_{2}\left(\pi \pi^{*}\right)$ states and between the $\mathrm{S}_{1}\left(n \pi^{*}\right)$ and $\mathrm{T}_{1}\left(n \pi^{*} / \pi \pi^{*}\right)$ states, respectively, whereas no significant SOC was found between $\mathrm{S}_{1}\left(n \pi^{*}\right)$ and $\mathrm{T}_{3}\left(\pi \pi^{*}\right)$. At the CASPT2 level, the singlet-triplet splittings $\left(\Delta E_{\mathrm{ST}}\right)$ between $\mathrm{S}_{1}$ and $\mathrm{T}_{1}$ and $\mathrm{S}_{1}$ and $\mathrm{T}_{2}$ are $0.53 \mathrm{eV}$ and $0.07 \mathrm{eV}$, respectively. Considering that an efficient population transfer from singlet to triplet states requires small energy gaps, the $\mathrm{S}_{1}\left(n \pi^{*}\right) / \mathrm{T}_{2}\left(\pi \pi^{*}\right)$ ISC mentioned above is expected to be the most viable mechanism 


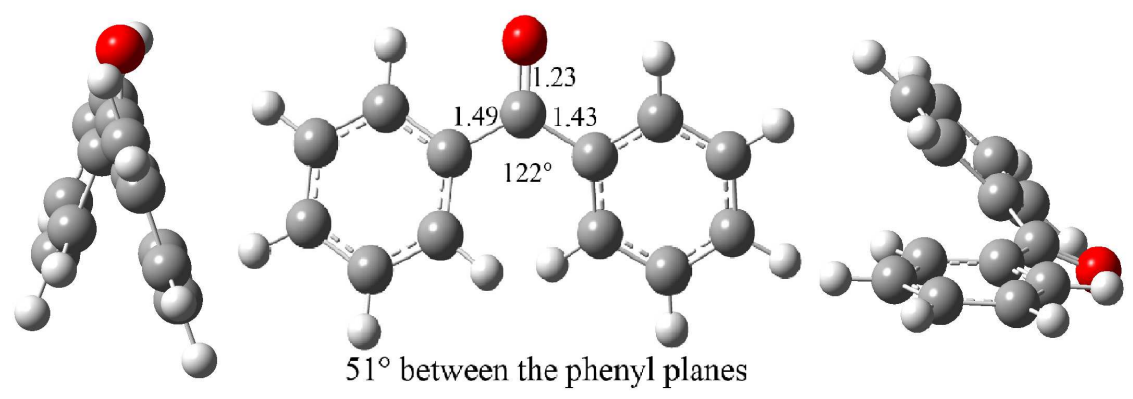

Fig. 4 Lowest-energy structure of the $\mathrm{S}_{2}\left(\pi \pi^{*}\right)$ excited state obtained at the end of the MEP computed at the CASSCF(12/11)/ANO-L-VDZP level of theory.

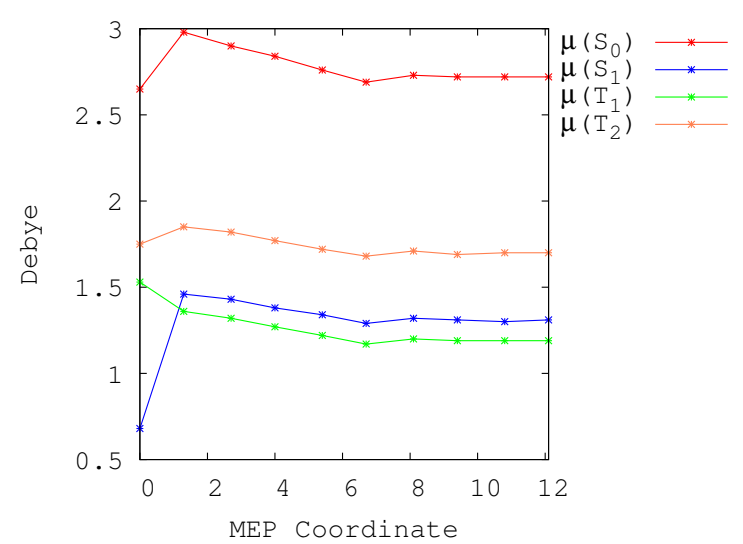

Fig. $6 \mathrm{CASSCF}(12 / 11)$ dipole moments of the ground and lowest-lying excited state along the MEP on the PES of $\mathrm{S}_{1}\left(n \pi^{*}\right)$.

for populating the triplet state in the molecule, out of the three proposed mechanisms. To gain more insight on the relative importance of $S_{1}$ in those three possible mechanisms, we pursue our analysis along the MEP. As can be seen in Figure 5, the energy difference between $\mathrm{S}_{1}$ and $\mathrm{T}_{1}$ can decrease down to $\sim 0.25 \mathrm{eV}$, which could allow a population transfer from $\mathrm{S}_{1}$ to $\mathrm{T}_{1}$. However, the SOC with the $T_{2}$ state, which also runs close in energy to $S_{1}$, is much larger than the one with $\mathrm{T}_{1}$. In particular, the computed $\mathrm{S}_{1}\left(n \pi^{*}\right) / \mathrm{T}_{2}\left(\pi \pi^{*}\right)$ and $\mathrm{S}_{1}\left(n \pi^{*}\right) / \mathrm{T}_{1}\left(n \pi^{*} / \pi \pi^{*}\right)$ SOC magnitudes for the MEP points that follows the FC region are around 59 and $22 \mathrm{~cm}^{-1}$, respectively. These findings point to the indirect mechanism in the gas-phase, in which an intermediate $\mathrm{T}_{2}$ state is initially populated, instead of a direct population of the lowest-lying $\mathrm{T}_{1}$ state.

In order to estimate the solvent effects and, particularly, the plausability of the direct $\mathrm{T}_{1}$ population in solu- tion, an analysis of the dipole moment $(\mu)$ values of the $\mathrm{S}_{0}, \mathrm{~S}_{1}, \mathrm{~T}_{1}$, and $\mathrm{T}_{2}$ along the MEP is considered. Figure 6 compiles the $\mu$ values computed with CASSCF. At the FC region, a more important blueshift is estimated for $\mathrm{S}_{1}$ than for $\mathrm{T}_{1}$ and $\mathrm{T}_{2}$. It implies that the $\mathrm{S}_{1} / \mathrm{T}_{2}$ CI may appear slightly displaced from the $\mathrm{FC}$ and also that the $\mathrm{S}_{1}-\mathrm{T}_{1}$ energy separation may be larger, hence unfavouring the direct population of $\mathrm{T}_{1}$. Along the MEP, the $\mu$ values of $S_{1}$ and $T_{1}$ are similar to each other and lower than those of $\mathrm{T}_{2}$. Thus, a less important blueshift can be expected for $\mathrm{T}_{2}$, thus approaching the $\mathrm{S}_{1}$ and $\mathrm{T}_{2}$ PESs, whereas the energy separation between the PESs of $\mathrm{S}_{1}$ and $T_{1}$ is estimated to remain as in the gas phase. Nevertheless, these $\mu$ differences and the estimated blueshifts are small, which may explain the fact that experimentalists do not observe major differences when solvents with different polarities are used. ${ }^{2}$

On the basis of the present findings, it can be predicted for the gas phase and solution, in general, that after irradiation to the $S_{1}$ state, the $S_{1}$ and $T_{2}$ cross in the $\mathrm{FC}$ region and both states evolve very close in terms of energy along the $\mathrm{S}_{1} \mathrm{MEP}$ (see figure 5) with a large probability of population transfer due to the high SOC. On the other hand, $\mathrm{T}_{1}$ is shown to have a relatively larger energy separation and lower SOC interaction.

The relaxation pathway involving fluorescence from the $\mathrm{S}_{1}\left(n \pi^{*}\right)$ state is not likely to be efficient since, along the MEP of the $S_{1}$ excited state, the conditions for a fast singlet to triplet population transfer are fulfilled, i.e., (i) small energy gap with the lowest-lying $\mathrm{T}_{2}$ state and (ii) large SOC magnitudes. This is in agreement with the experimental observation that the benzophenone molecule exhibits mainly phosphorescence (with an associated quantum yield of $84 \%),{ }^{48,49}$ and not fluorescence. Note that the computed CASPT2 vertical emission energy from the $\mathrm{S}_{1}\left(n \pi^{*}\right)_{\text {min }}$ state is $2.34 \mathrm{eV}$. 


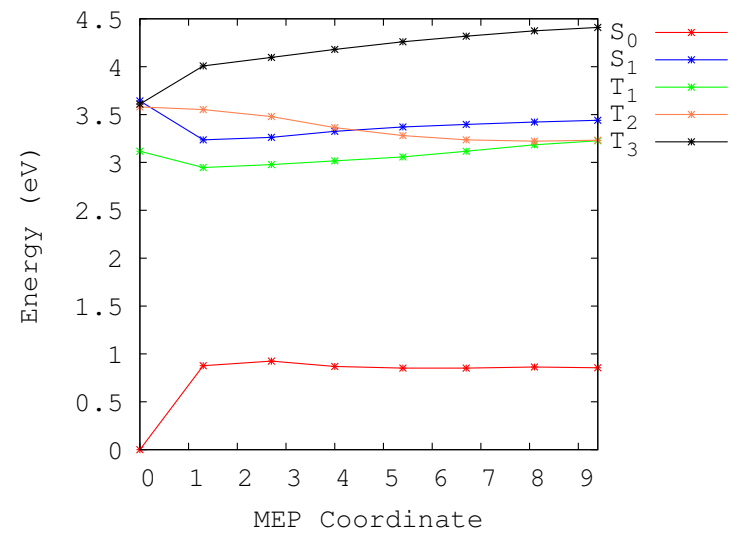

Fig. 7 CASPT2(12/11)//CASSCF(12/11) energies of the ground and lowest-lying singlet and triplet excited states of benzophenone along the MEP on the PES of the $\mathrm{T}_{2}\left(\pi \pi^{*}\right)$ state from the $\mathrm{S}_{1}\left(n \pi^{*}\right) / \mathrm{T}_{2}\left(\pi \pi^{*}\right) \mathrm{STC}$ at the $\mathrm{FC}$ region toward the $\mathrm{T}_{2}\left(\pi \pi^{*}\right) / \mathrm{T}_{1}\left(n \pi^{*} / \pi \pi^{*}\right)$ CI.

4.2.2 Triplet manifold. The MEP on the PES of $\mathrm{S}_{1}\left(n \pi^{*}\right)$ from the $\mathrm{FC}$ region toward its minimum structure indicates that the $\mathrm{T}_{2}\left(\pi \pi^{*}\right)$ state of benzophenone can be efficiently populated via the $\mathrm{S}_{1}\left(n \pi^{*}\right) / \mathrm{T}_{2}\left(\pi \pi^{*}\right)$ ISC process described above. Figure 7 shows the subsequest evolution of the triplet manifold by the MEP computation of the $\mathrm{T}_{2}$ state from the crossing region with $\mathrm{S}_{1}$. It can be seen that the MEP on the $\mathrm{T}_{2}$ PES leads initially to a re-crossing point with the $\mathrm{S}_{1}\left(n \pi^{*}\right)$ excited state and finally to the $\mathrm{T}_{2}\left(\pi \pi^{*}\right) / \mathrm{T}_{1}\left(n \pi^{*} / \pi \pi^{*}\right)$ CI. Hence, two different relaxation pathways can be assumed at this point:

- via a $\mathrm{T}_{2}\left(\pi \pi^{*}\right) / \mathrm{S}_{1}\left(n \pi^{*}\right)$ re-crossing favouring the repopulation of the $\mathrm{S}_{1}\left(n \pi^{*}\right)$ state

- via a $\mathrm{T}_{2}\left(\pi \pi^{*}\right) / \mathrm{T}_{1}\left(n \pi^{*} / \pi \pi^{*}\right)$ CI leading to the phosphorescent $\mathrm{T}_{1}$ state

We start by discussing the ISC re-crossing channel. The computed SOC magnitude between the $\mathrm{T}_{2}$ and $\mathrm{S}_{1}$ states at the re-crossing point is relatively large: $47 \mathrm{~cm}^{-1}$. This suggests that part of the population may be transferred back to the $\mathrm{S}_{1}$ state via an efficient ISC process. From the S-T re-crossing point, a MEP, computed on the PES of the $\mathrm{S}_{1}$ state with CASPT2//CASSCF (see Figure 8 ), leads directly to the $\mathrm{S}_{1}\left(n \pi^{*}\right)_{\min }$. The behavior of the $\mathrm{S}_{1}$ and $\mathrm{T}_{2}$ excited states along the MEPs displayed in Figures 7 and 8 confirms the aforementioned hypothesis of a strong link between both states in the photoexcited benzophenone. Hence, taking into consideration the small energy difference (which becomes even smaller assuming a polar solvent) and high SOCs between $\mathrm{S}_{1}$ and $\mathrm{T}_{2}$

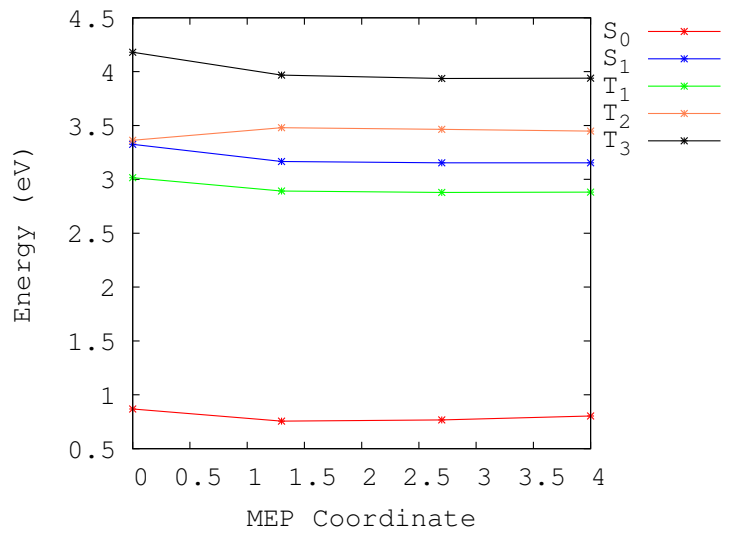

Fig. 8 CASPT2(12/11)//CASSCF(12/11) energies of the ground and lowest-lying singlet and triplet excited states of benzophenone along the MEP on the PES of the $\mathrm{S}_{1}\left(n \pi^{*}\right)$ state from the $\mathrm{T}_{2}\left(\pi \pi^{*}\right) / \mathrm{S}_{1}\left(n \pi^{*}\right)$ re-crossing point toward $\mathrm{S}_{1}\left(n \pi^{*}\right)_{\min }$.

(around $59 \mathrm{~cm}^{-1}$ along the $\mathrm{S}_{1} \mathrm{MEP}$ ), the population lost with the re-crossing may be transferred back and forth to the $T_{2}$ state via several re-crossing processes, and finally the wave-packet may be driven toward the $T_{2} / T_{1}$ CI, which appears at lower energies compared to the PES of $\mathrm{S}_{1}$ and other points on the $\mathrm{T}_{2}$ surface. It is worth mentioning here that the presence of the $\mathrm{T}_{2} / \mathrm{S}_{1}$ re-crossings (Figure 7) may be related to the E-type delayed fluorescence reported experimentally by Brown and Singer. ${ }^{50}$ Briefly, after the low fluorescence has ended, the spectrum does not show only a phosporescence spectral distribution but also some residual intensity (fluorescence) that decay at the same rate as the phosphorescence.

We finish the discussion by commenting on the second proposed relaxation mechanism from the $\mathrm{T}_{2}$ state. The $\mathrm{T}_{2}\left(\pi \pi^{*}\right) / \mathrm{T}_{1}\left(n \pi^{*} / \pi \pi^{*}\right)$ CI is computed to be adiabatically at $3.22 \mathrm{eV}$ above the $\mathrm{S}_{0}$ state at the CASPT2 level, favouring the population switch toward the $\mathrm{T}_{1}$ state. At the same level of theory, the energy splitting between the two states $\left(\Delta E_{\mathrm{CI}}\right)$ is computed to be $0.004 \mathrm{eV}$. The geometry of $\mathrm{T}_{2}$ at the CI shows a coplanarity of one of the phenyl rings with the carbonyl group, a $\mathrm{CO}$ bond distance of $1.35 \AA$, and a dihedral angle of $45^{\circ}$ between the two phenyl planes. From the $\mathrm{T}_{2}\left(\pi \pi^{*}\right) / \mathrm{T}_{1} \mathrm{CI}$, the MEP on the $\mathrm{T}_{1}\left(n \pi^{*} / \pi \pi^{*}\right)$ PES leads directly to the $\mathrm{T}_{1}$ state minimum, $\mathrm{T}_{1}\left(n \pi^{*} / \pi \pi^{*}\right)_{\min }$ (see Figure 9), which is placed adiabatically at $2.85 \mathrm{eV}$ in agreement with the band origin observed experimentally at $3.00 \mathrm{eV}{ }^{3}$ The computed vertical emission energy (phosphorescence) from the $\mathrm{T}_{1}\left(n \pi^{*} / \pi \pi^{*}\right)_{\min }$ equilibrium structure is $2.19 \mathrm{eV}$. Therefore, we conclude that the $\mathrm{T}_{2}\left(\pi \pi^{*}\right)$ state plays a 


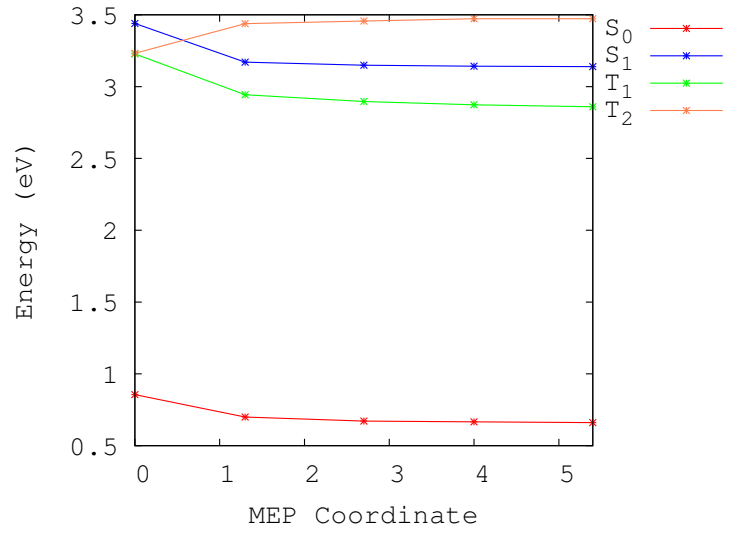

Fig. 9 CASPT2(12/11)//CASSCF(12/11) energies of the ground and lowest-lying singlet and triplet excited states of benzophenone along the MEP on the PES of the $\mathrm{T}_{1}\left(n \pi^{*} / \pi \pi^{*}\right)$ state from the $\mathrm{T}_{2}\left(\pi \pi^{*}\right) / \mathrm{T}_{1}\left(n \pi^{*} / \pi \pi^{*}\right)$ CI toward $\mathrm{T}_{1}\left(n \pi^{*} / \pi \pi^{*}\right)_{\min }$.

crucial role in the fast ISC in benzophenone, as in other related conjugated ketones and aldehydes. ${ }^{51,52}$

\section{Conclusion}

In this work, the CASPT2//CASSCF approach with the double- $\zeta$ plus polarization ANO-L basis set was used to revisit the photophysics of benzophenone, by means of computations of MEPs and the determination of relevant energy minima and CIs. The obtained results are in good agreement with the experimental findings that suggest a two-step kinetic model $\left(\mathrm{S}_{1} \rightarrow \mathrm{IS} \rightarrow \mathrm{T}_{1}\right)$ relaxation mechanism of the $S_{1}$ excited state ${ }^{2}$, and we attribute the intermediate state to $\mathrm{T}_{2}\left(\pi \pi^{*}\right)$. Moreover, the results allow rationalizing the low quantum yield of fluorescence of benzophenone and suggest an explanation for the Etype delayed fluorescent emission signal observed experimentally. ${ }^{50}$ The main relaxation mechanisms from the $\mathrm{S}_{1}\left(n \pi^{*}\right)$ excited state are shown schematically in Figure 10. After populating the $\mathrm{S}_{1}\left(n \pi^{*}\right)$ state in the FC region, the $\mathrm{S}_{1}\left(n \pi^{*}\right)_{\text {min }}$ point, which lies $3.15 \mathrm{eV}$ above $\mathrm{S}_{0}$, can be reached. However, this path is not expected to be predominant since a strong coupling between the $\mathrm{S}_{1}\left(n \pi^{*}\right)$ and $\mathrm{T}_{2}\left(\pi \pi^{*}\right)$ states is computed already in the $\mathrm{FC}$ region, which may result in an efficient population transfer to the $\mathrm{T}_{2}$ state in between the $\mathrm{FC}$ region and $\mathrm{S}_{1}\left(n \pi^{*}\right)_{\text {min }}$. Favorable intersystem re-crossings leading to the $\mathrm{S}_{1}$ state are found which may bring back population to the $\mathrm{S}_{1}$ state and that explains the observable delayed fluorescence signal. ${ }^{50}$ Eventually, the system may further de-

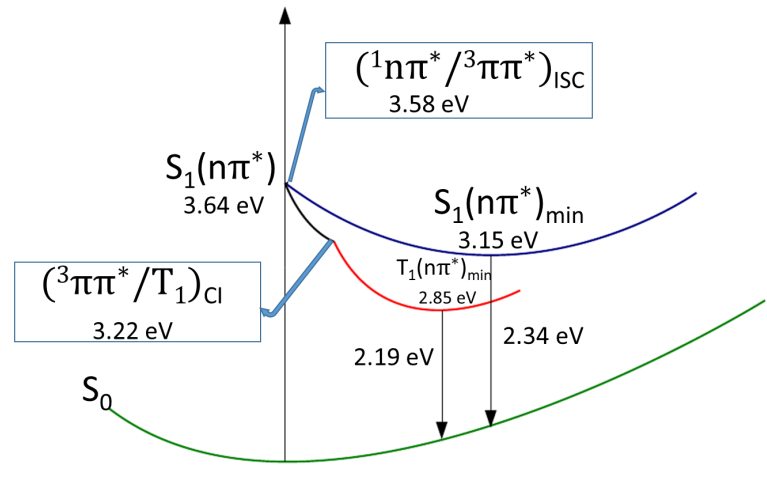

Fig. 10 Schematic representation of the main potential decay mechanisms of benzophenone from the lowest-lying singlet $n \pi^{*}$ excited state.

cay along the $\mathrm{T}_{2}\left(\pi \pi^{*}\right)$ state toward the $\mathrm{T}_{2} / \mathrm{T}_{1} \mathrm{CI}$, which may funnel the energy to the lowest-lying triplet state $\mathrm{T}_{1}\left(n \pi^{*} / \pi \pi^{*}\right)$. Finally, the molecule may arrive in a barrierless manner to the equilibrium structure of the $T_{1}$ state, $\mathrm{T}_{1}\left(n \pi^{*} / \pi \pi^{*}\right)_{\text {min }}$, at $2.85 \mathrm{eV}$ from where it efficiently phosphoresces. We note that a computational chemistry perspective of this work could consist in computing with quantum dynamics the ISC rate in benzophenone, which would be a highly demanding task owing the complexity of the involved electronic states.

\section{Acknowledgments}

Helpful discussions with Prof. Manuela Merchán are acknowledged. The research was supported by the EMTCCM master program and by the Project CTQ201014892 of the Spanish MEC/FEDER. R.W.A.H. acknowledges the Zernike Institute for Advanced Materials ("Dieptestrategie program") for financial support. D.R.S. thanks the "Juan de la Cierva" program of the Spanish MINECO (Ref. JCI-2012-13431).

\section{References}

1 R. Katoh, M. Kotani, Y. Hirata and T. Okada, Chem. Phys. Lett., 1997, 264, 631-635.

2 S. Alö̈se, C. Ruckebusch, L. Blanchet, J. Réhault, G. Buntix and J.-P. Huvenne, J. Phys. Chem. A, 2008, 112, 224-231.

3 N. Ohmori, T. Suzuki and M. Ito, J. Phys. Chem., 1988, 92, 1086-1093.

4 P. F. McGarry, C. E. Doubleday, C.-H. Wu, H. A. Staab and N. J. Turro, J. Photochem. Photobiol. A: Chem., 1994, 77, 109-117.

5 M. El-Sayed, Acc. Chem. Res., 1968, 1, 8-16.

6 S. Yabumoto, S. Sato and H. Hamaguchi, Chem. Phys. Lett., 2005, 416, 100-103. 
7 W. Fuß, S. Cochbrunner, A. M. Müller, T. Chikarski, W. E. Schmid and S. A. Trushin, Chem. Phys., 1998, 232, 161-174.

8 M. A. Robb, M. Garavelli, M. Olivucci and F. Bernardi, Rev. Comput. Chem., 2000, 15, 87-146.

9 M. Olivucci, Theoretical and Computational Chemistry, Elsevier, Amsterdam, 2005.

10 A. Giussani, J. Segarra-Martí, D. Roca-Sanjuán and M. Merchán, Top. Curr. Chem., 2013, DOI: 10.1007/128_2013_501.

11 M. Robb, F. Bernardi and M. Olivucci, Pure \& Appl. Chem., 1995, 67, 783-789.

12 M. El-Sayed and R. Leyerle, J. Chem. Phys., 1975, 62, 15791580 .

13 M. Batley and D. R. Kearns, Chem. Phys. Lett., 1968, 2, 423425.

14 Y. Matsushita, Y. Kajii and K. Obi, J. Phys. Chem., 1992, 96, $4455-4458$.

15 H. Miyasaka, K. Morita, K. Kamada and N. Mataga, Bull. Chem. Soc. Jpn., 1990, 63, 3385-3397.

16 F. Weigend and R. Ahlrichs, Phys. Chem. Chem. Phys., 2005, 7, 3297-3305.

17 TURBOMOLE, V6.4 2012, a development of University of Karlsruhe and Forschungszentrum Karlsruhe GmbH, 19892007, TURBOMOLE GmbH, since 2007; available from http://www.turbomole.com.

18 J. P. Perdew, M. Ernzerhof and K. Burke, J. Phys. Chem., 1996, 105, 9982-9985.

19 C. Adamo and V. Barone, J. Chem. Phys., 1999, 110, 61586170 .

20 B. O. Roos In, Advances in Chemical Physics; Ab Initio Methods in Quantum Chemistry II, K. P. Lawley, Ed., Wiley \& Sons Ltd., Chichester, England, 1987, pp. 399-445.

21 B. O. Roos, P. R. Taylor and P. E. M. Siegbahn, Chem. Phys., 1980, 48, 157-173.

22 P.-O. Widmark, P.-A. Malmqvist and B. O. Roos, Theor. Chim. Acta, 1991, 77, 291-306.

23 P.-O. Widmark, B. Joakim, B. J. Persson and B. O. Roos, Theor. Chim. Acta, 1991, 79, 419-432.

24 K. Andersson, F. Aquilante, A. Bernhardsson, M. R. A. Blomberg, D. L. Cooper, M. Cossi, A. Devarajan, L. De Vico, N. Ferré, M. P. Fülscher, A. Gaenko, L. Gagliardi, G. Ghigo, C. de Graaf, B. A. Heß, D. Hagberg, A. Holt, G. Karlström, J. W. Krogh, R. Lindh, P.-Å. Malmqvist, P. Neogrády, J. Olsen, T. B. Pedersen, M. Pitonak, J. Raab, M. Reiher, B. O. Roos, U. Ryde, I. Schapiro, B. Schimmelpfennig, L. Seijo, L. SerranoAndrés, P. E. M. Siegbahn, J. Stålring, T. Thorsteinsson, S. Vancoillie, V. Veryazov, P. O. Widmark and A. Wolf, MOLCAS version 7.6, Lund University, Sweden.

25 L. De Vico, M. Olivucci and R. Lindh, J. Chem. Theory Comput., 2005, 1, 1029-1037.

26 P.-A. Malmqvist, B. O. Roos and B. Schimmelpfennig, Chem. Phys. Lett., 2002, 357, 230-240.
27 B. O. Roos and P.-Å. Malmqvist, Phys. Chem. Chem. Phys., 2004, 6, 2919-2927.

28 B. A. Heß, C. M. Marian, U. Wahlgren and O. Gropen, Chem. Phys. Lett., 1996, 251, 365-371.

29 O. Christiansen, J. Gauss and B. Schimmelpfenning, Phys. Chem. Chem. Phys., 2000, 2, 965-971.

30 K. Andersson, P.-Å. Malmqvist, B. O. Roos, A. J. Sadlej and K. Wolinski, J. Phys. Chem., 1990, 94, 5483-5488.

31 K. Andersson, P.-A. Malmqvist and B. O. Roos, J. Chem. Phys., 1992, 96, 1218-1226.

32 N. Forsberg and P.-Å. Malmqvist, Chem. Phys. Lett., 1997, 274, 196-204.

33 G. Ghigo, B. O. Roos and P.-Å. Malmqvist, Chem. Phys. Lett., 2004, 396, 142-149.

34 P.-Å. Malmqvist, K. Pierloot, A. R. M. Shahi, C. J. Cramer and L. Gagliardi, J. Chem. Phys., 2008, 128, 204109.

35 J. Olsen, B. O. Roos, P. Jorgensen and H. Jensen, J. Chem. Phys., 1988, 89, 2185-2193.

36 M. Valiev, E. Bylaska, N. Govind, K. Kowalski, T. Straatsma, H. van Dam, D. Wang, J. Nieplocha, T. W. E. Apra and W. de Jong, Comput. Phys. Commun., 2010, 181, 1477-1489.

37 Dalton, a molecular electronic structure program, Release Dalton2011 (2011), see http://daltonprogram.org.

38 P. Sett, T. Misra, S. Chattopadhyay, A. De and P. Mallick, Vibrational Spectroscopy, 2007, 44, 331-342.

39 R. Hoffmann and J. Swenson, J. Phys. Chem., 1969, 74, 415420.

40 E. B. Fleischer, N. Sung and S. Hawkinson, J. Phys. Chem., 1968, 72, 4311-4312.

41 R. M. Hochstrasser and L. J. Noe, Journal of Molecular Spectroscopy, 1971, 38, 175-180.

42 J. W. Barker and L. J. Noe, J. Phys. Chem., 1972, 57, 30353041.

43 T. Hiroto, I. Tetsuji and K. Kohichi, Phys. Chem. Chem. Phys., 2009, 11, 6008-6014.

44 L. Wendell, J. Org. Chem., 1965, 31, 1045-1050.

45 K. K. Chin, A. Natarajan, M. N. Gard, L. M. Campos, H. Shepherd, E. Johansson and M. A. Garcia-Garibay, Chem. Commun., 2007, 4266-4268.

46 G. Spighi, M.-A. Gaveau, J.-M. Mestdagh, L. Poisson and B. Soep, Phys. Chem. Chem. Phys., 2014, 16, 9610-9618.

47 E. Dumont and A. Monari, J. Phys. Chem. Lett., 2013, 4, 41194124.

48 E. H. Gilmore, G. E. Gibson and D. S. McClure, J. Chem. Phys., 1952, 20, 829-836.

49 E. H. Gilmore, G. E. Gibson and D. S. McClure, J. Chem. Phys., 1955, 23, 399.

50 R. Brown and L. Singer, Chem. Phys. Lett., 1972, 14, 193-195.

51 M. Huix-Rotlland, D. Siri and Ferré, Phys. Chem. Chem. Phys., 2013, 15, 19293-19300.

52 Q. Ou and J. E. Subotnik, J. Phys. Chem. C, 2013, 117, 19839 19849. 


\section{Table of contents:}

\section{Artwork:}

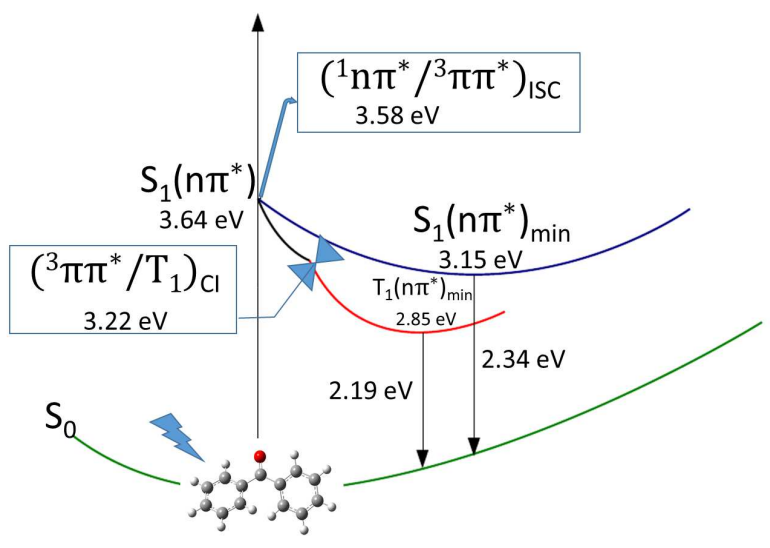

\section{Synopsis:}

The dominant triplet population mechanism in benzophenone is determined from wave function based calculations, and involves the $\mathrm{T}_{2}\left(\pi \pi^{*}\right)$ state as an intermediate between $\mathrm{S}_{1}$ and $\mathrm{T}_{1}$. 